\title{
Relation entre la fermeté de la mangue fraîche et la teneur en amidon de la pulpe
}

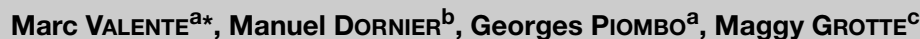

a Cirad-Amis, Programme Agro-Alimentaire, TA40/15, 73 rue J.F. Breton, 34398 Montpellier, Cedex 5, France marc.valente@cirad.fr

${ }^{b}$ Ensia-Siarc, 1101 avenue Agropolis, CS 24501, 34093 Montpellier Cedex 5, France

c INRA - SQPOV, Site Agroparc, Domaine Saint-Paul, 84914 Avignon Cedex 9, France

* Correspondance et tirés à part

Reçu le 21 juin 2004

Accepté le 27 septembre 2004

Fruits, 2004, vol. 59, p. 399-410 (C) 2005 Cirad/EDP Sciences All rights reserved

DOI: $10.1051 /$ fruits:2005002

RESUMEN ESPAÑoL, p. 410

\section{Mango fruit firmness and pulp starch content.}

Abstract - Introduction. Fruit firmness and pulp starch content are two physicochemical traits of great interest for a climacteric fruit such as mango. These two indicators have long been recognised as relevant to the study of fruit maturity at harvest and during ripening and storage. However, very few works have been conducted on the relationship between starch content, one of the major components of mango pulp at harvest, and whole-fruit firmness. Materials and methods. Firmness evaluation of the whole fruit was carried out using both a destructive mechanical method and a nondestructive acoustic impulse response method. Penetration force $F 5(\mathrm{~N})$ was measured at $5.5 \mathrm{~mm}$ depth when a cylindrical plunger (diameter: $6.35 \mathrm{~mm}$ ) with a conical tip penetrated unpeeled fruit at a speed of $3 \mathrm{~mm} \cdot \mathrm{s}^{-1}$. A global elasticity coefficient, $E(\mathrm{~Pa})$, was estimated from the greatest resonance frequency of the whole fruit, obtained by the acoustic impulse response technique. Starch content was determined on a representative sample of whole pulp of each fruit. Analysis of glucose released by starch hydrolysis was carried out by ionic chromatography. Experiments were performed on 133 fruits (cvs. Keitt and Amelie) covering a wide range of maturity stages. Results and discussion Starch content was highly correlated with the two firmness parameters $E$ and $F 5$. The best correlation coefficients were obtained for Keitt mangoes ( $r=0.89$ for $E ; r=0.88$ for $F 5)$ and for all the fruit of the two varieties ( $r=0.83$ for $E ; r=0.76$ for $\mathrm{F} 5$ ). On the contrary, starch content was poorly correlated with penetration force $F 5(r=0.60)$ for Amelie mangoes, the elasticity coefficient, $E$, remaining, however, highly correlated with starch content $(r=0.75)$. Conclusion. The acoustic impulse response technique appears to be a useful tool for predicting mango fruit shelf life by simultaneously allowing whole-fruit firmness estimation and pulp starch content evaluation. The correlation level obtained between the elasticity coefficient, $E$, and pulp starch content depends on the mango variety. In the case of a high correlation level such as for Keitt mangoes, the acoustic impulse technique could give information on the starch degradation level in mango as reliable as those obtained by using a destructive iodine test.

France / Mangifera indica / fruits / firmness / starch / nondestructive methods / measurement / penetrometers

\section{Relation entre la fermeté de la mangue fraîche et la teneur en amidon de la pulpe.}

Résumé - Introduction. La fermeté du fruit et la teneur en amidon de la pulpe sont deux critères physico-chimiques d'un grand intérêt pour un fruit climactérique comme la mangue. Ils constituent deux bons indicateurs de la maturité des fruits à la cueillette, et de leur degré de maturation en cours de conservation. À notre connaissance, peu d'études font le lien entre la teneur en amidon de la pulpe, composé majeur de la mangue au stade de la cueillette, et la fermeté du fruit. Matériel et méthodes. Deux méthodes d'évaluation de la fermeté du fruit entier ont été mises en œuvre : une méthode pénétrométrique destructive permettant d'accéder à la force développée pour pénétrer le fruit jusqu'à une profondeur de $5,5 \mathrm{~mm}(F 5)$ et une méthode acoustique impulsionnelle non destructive permettant de déterminer un coefficient global d'élasticité $(E)$ du fruit. La teneur en amidon a été déterminée sur un échantillon, représentatif de la pulpe entière du fruit, traité par hydrolyse acide puis enzymatique. Le glucose libéré a été dosé par chromatographie ionique. Les essais ont été réalisés sur 133 fruits au total, appartenant à deux populations de mangues des variétés Keitt et Amélie et couvrant une gamme étendue de maturité. Résultats et discussion. La teneur en amidon de la pulpe se révèle bien corrélée aux deux paramètres de fermeté $F 5$ et $E$ mesurés sur fruit entier. Les meilleurs coefficients de corrélation ont été obtenus pour la population de mangues Keitt $(r=0,89$ pour $E ; r=0,88$ pour $F 5$ ) et pour la population rassemblant l'ensemble des fruits des deux variétés $(r=0,83$ pour $E ; r=$ 0,76 pour $F 5$ ). La variété Amélie se distingue par un faible coefficient de corrélation entre la teneur en amidon et la fermeté pénétrométrique $F 5(r=0,60)$, le coefficient d'élasticité $E$ demeurant encore relativement bien corrélé à la teneur en amidon $(r=0,75)$. Conclusion. La méthode acoustique impulsionnelle peut constituer un outil intéressant capable d'apprécier, de manière non destructive, le potentiel de conservation des fruits par l'évaluation concomitante de la fermeté globale du fruit et de la teneur résiduelle en amidon de sa pulpe. Le coefficient de corrélation observé entre le coefficient d'élasticité $E$ et la teneur en amidon diffère selon la variété de mangue. Lorsque ce coefficient de corrélation est élevé, la technique acoustique impulsionnelle peut donner, indirectement, d'aussi bonnes informations sur le niveau de dégradation de l'amidon dans le fruit en cours de conservation qu'une méthode destructive comme celle du test à l'iode.

France / Mangifera indica / fruits / fermeté / amidon / méthode non destructive / mesure / pénétromètre 


\section{Introduction}

Les échanges mondiaux relatifs au commerce de la mangue fraîche représentent seulement $2,5 \%$ de la production mondiale de ce fruit. Le développement du commerce en frais de la mangue est freiné par des contraintes techniques, liées au caractère très périssable de ce fruit et à sa physiologie [1]. Au premier rang des difficultés rencontrées lors de la commercialisation des fruits, figurent le maintien de la qualité des fruits au cours du transport par voie maritime, sur de longues distances, et surtout l'hétérogénéité de maturité des lots de fruits mis en marché [2, 3].

L'hétérogénéité de maturité des fruits est liée, en grande partie, à la variabilité du stade physiologique de développement des fruits au moment de leur cueillette, ainsi qu'aux difficultés d'évaluer de façon objective le stade optimal de cueillette particulièrement important pour un fruit climactérique comme la mangue. Selon Pruthi [4], le stade de maturité physiologique des mangues à la cueillette influence leur aptitude au transport, à la conservation, au mûrissement et donc la qualité finale des fruits proposés aux consommateurs. De plus la présence de quelques fruits de maturité avancée peut induire puis accélérer le mûrissement de la totalité du lot.

Différents indices de maturité ont été rapportés dans la littérature [3-5] afin de repérer le stade physiologique optimal de cueillette des mangues assurant une expression maximale des qualités organoleptiques (saveur, flaveur, texture) de la pulpe après mûrissement des fruits. À côté de méthodes générales basées sur des critères physiologiques (âge du fruit depuis la floraison ou la nouaison), parfois combinés à des données climatiques (sommes thermiques, degrés-jour), ont été proposées des méthodes plus spécifiques fondées sur :

- des aspects visuels du fruit (caractéristiques morphologiques, couleur de la peau), - des critères physiques du fruit (poids, densité),

- des critères physico-chimiques de la pulpe (extrait sec soluble, amidon, sucres totaux, acidité, couleur, fermeté).

Compte tenu des différences importantes existant entre les nombreux cultivars de mangue, aucun de ces indices de maturité, utilisés de façon isolée, n'est valide pour l'ensemble des variétés commerciales [4]. Dans la pratique, l'expérience des producteurs conduit à utiliser deux ou trois de ces indices dont la combinaison, pour une variété et une région de production données, facilite la détermination du stade de cueillette [2].

L'amidon constitue un composé majeur de la pulpe du fruit au stade de la cueillette. Son accumulation, lente en début de croissance du fruit, s'accélère sensiblement peu avant la maturité physiologique du fruit sur l'arbre [6], pour atteindre des teneurs variant de $(4,2$ à 10,6) \% [7, 8]. Après cueillette, l'hydrolyse enzymatique de l'amidon est élevée au moment de la crise climactérique du fruit, puis elle décroît fortement à l'approche de la pleine maturité. À ce stade, la pulpe amollie du fruit présente des teneurs résiduelles en amidon variant de $(0,1$ à 0,4$) \%$ selon la variété et la température de stockage [9].

Ces variations marquées de la teneur en amidon dans la pulpe ont conduit certains auteurs à proposer l'utilisation de la teneur en amidon ou du ratio [amidon / acides organiques] comme critères de maturité de ce fruit [10]. Mais ces travaux ont trouvé, d'un point de vue pratique, peu d'échos en raison de la lourdeur du protocole opératoire à mettre en œuvre pour l'analyse en laboratoire de l'amidon. Les techniques d'estimation rapide du taux de régression de l'amidon, basées sur un test à l'iode, ont été récemment utilisées par Rocha et al. [11] pour déterminer sur le terrain le stade de maturité de la mangue. L'indice de régression de l'amidon, noté sur une échelle de 0 à 6 , est très significativement corrélé à la coloration de la pulpe $(r=0,95)$ perçue comme un bon indicateur de la maturité du fruit. Cependant, ces tests à l'iode, basés sur une évaluation visuelle de l'aire des zones colorées plus ou moins dispersées, sont relativement subjectifs. Cela est en particulier lié à la stratégie utilisée par chaque opérateur pour attribuer à l'échantillon un indice de régression d'amidon, en se référant à un code amidon comportant plusieurs indices de référence. À titre d'exemple, Peirs et al. [12] ont étudié les principales causes d'erreur d'estimation du niveau réel de régression de 
l'amidon pour la pomme, dont le code amidon comporte 10 indices de référence.

L'estimation rapide de la teneur en amidon de la mangue au moyen de méthodes physiques non destructives a assez peu fait l'objet d'études. Selon Saranwong et al. [13], la spectrométrie proche infrarouge pourrait être utilisée pour estimer, de manière non destructive, les teneurs en matières sèches et en amidon des mangues au stade de la cueillette.

De nombreux travaux ont été consacrés à l'étude des différentes techniques d'évaluation de la fermeté de la mangue et au suivi de son évolution au cours de la maturation sur l'arbre [14] et après cueillette [15, 16]. La fermeté pénétrométrique, mesurée au moyen d'un test de poinçonnage du fruit, demeure la mesure de fermeté la plus utilisée, en raison de sa simplicité et de sa possible réalisation au champ. Elle représente la force maximale de pénétration d'un poinçon dans le fruit, épluché ou non, jusqu'à une profondeur prédéfinie. Elle est généralement exprimée en newton $(\mathrm{N})$, en kilogramme-force (kgf) ou en pound-force (lbf), mais certaines données de fermeté pénétrométrique de la mangue relevées dans la littérature sont exprimées en $\mathrm{kg} \cdot \mathrm{cm}^{-2}$, le diamètre du poinçon n'étant par ailleurs pas toujours précisé. À défaut de standardisation de cette méthode, définissant avec précision le diamètre et la forme du poinçon, la vitesse et la profondeur de pénétration dans la pulpe ainsi que le nombre de poinçonnages par fruit, les valeurs obtenues par les différents auteurs peuvent difficilement faire l'objet de comparaison [17].

Des méthodes physiques d'évaluation non destructives de la fermeté de la mangue, donnant accès à des propriétés physiques intrinsèques du matériau (module d'élasticité, vitesse de propagation du son, amortissement) sont en cours de développement [16, 18-20]. Dans la présente étude, deux méthodes d'évaluation de la fermeté ont été mises en œuvre : une méthode pénétrométrique destructive et une méthode acoustique non destructive.

L'objectif de nos travaux a été double :

- établir les relations existant entre la teneur en amidon de la pulpe et la fermeté du fruit, évaluée à l'aide des deux méthodes précitées,
- préciser la contribution de l'amidon à l'élaboration des propriétés de texture de la pulpe, sachant que cette contribution s'exprime de façon concomitante aux modifications de composition et de structure des parois cellulaires de la pulpe déjà identifiées comme responsables de l'amollissement de la pulpe, lors du mûrissement des mangues.

\section{Matériel et méthodes}

\subsection{Matériel végétal}

Les essais ont porté sur 133 fruits, appartenant à deux variétés commerciales Amélie et Keitt, en provenance de Côte d'Ivoire. Les fruits ont été sélectionnés de manière à couvrir une large gamme de maturité. L'approvisionnement s'est fait auprès d'importateurs.

À partir d'un lot unique de fruits de la variété Keitt livrés au laboratoire, deux lots expérimentaux ont été constitués. Le premier lot, homogène en maturité, composé de 40 fruits immatures à pulpe très ferme [fréquence de résonance comprise entre (600 et 700) Hz], a été placé en conservation à $13{ }^{\circ} \mathrm{C}$ et $90 \%$ d'humidité relative (HR). Dix fruits ont en été prélevés de manière aléatoire à $(5,12,19$ et 26) j de conservation. Le second lot, plus hétérogène en maturité, a été constitué de 44 fruits immatures et tournants ; ces fruits ont été analysés après 3 semaines de conservation à $13{ }^{\circ} \mathrm{C}$ et $90 \%$ HR.

À l'opposé, les fruits de variété Amélie ont été achetés à intervalles de temps réguliers, selon les besoins, sur une période de 1 mois environ et analysés le jour de leur achat ou le lendemain. Contrairement aux fruits de la variété Keitt, les 49 mangues Amélie analysées ne présentaient donc pas la garantie d'être issues d'une même provenance et d'une même récolte.

\subsection{Fermeté mécanique}

La fermeté pénétrométrique a été mesurée à l'aide d'un test de poinçonnage réalisé sur le fruit intact au niveau de la joue. Le 
poinçon qui a été utilisé est constitué d'un cylindre de $6,3 \mathrm{~mm}$ de diamètre à extrémité conique (angle de $60^{\circ}$ ) et monté sur un analyseur de texture TA-XT2 (Stable Micro System). Le poinçon pénètre le fruit non pelé à la vitesse de $3 \mathrm{~mm} \cdot \mathrm{s}^{-1}$ jusqu'à une profondeur de 5,5 mm [16]. L'analyseur de texture permet d'enregistrer la force de pénétration en fonction du déplacement. Par suite la fermeté pénétrométrique, notée $F 5$ et exprimée en Newton, représente la force mesurée à 5,5 mm de profondeur. Afin d'obtenir une bonne estimation de la fermeté globale du fruit, la valeur de $F 5$ retenue a été la moyenne d'au minimum huit mesures locales réalisées en des points uniformément répartis sur la surface des deux joues de la mangue [21].

\subsection{Fermeté acoustique}

La technique acoustique impulsionnelle utilisée pour évaluer, de manière non destructive, la fermeté globale de la mangue a été décrite précédemment [21]. Son principe repose sur l'identification de la fréquence de résonance de plus grande amplitude du fruit lorsque ce dernier est soumis à une excitation mécanique brève et énergétique. Le choc est assuré par la chute libre d'une bille en acier inoxydable de $12 \mathrm{~mm}$ de diamètre, d'une hauteur de $25 \mathrm{~cm}$ sur le centre de la joue du fruit posé sur un support. Un microphone, situé à quelques millimètres de la peau en position diamétralement opposée au point d'impact, enregistre la réponse acoustique consécutive au choc de la bille sur le fruit sur une durée de $25 \mathrm{~ms}$, sans tenir compte du rebond éventuel. La transformée de Fourier (FFT) du signal temporel permet d'obtenir le spectre de fréquence et de déterminer la fréquence de résonance de plus grande amplitude " $f$ ".

La fermeté acoustique du fruit est exprimée à l'aide d'un coefficient d'élasticité du fruit $E(\mathrm{~Pa})$, calculé à partir de la fréquence $f(\mathrm{~Hz})$, de la masse $M(\mathrm{~kg})$ et de la masse volumique $D\left(\mathrm{~kg} \cdot \mathrm{m}^{-3}\right)$, selon la relation $: E=f^{2} \cdot M^{2 / 3} \cdot D^{1 / 3}[22,23]$.

La valeur de la fréquence $f$, utilisée pour le calcul de $E$, représente la moyenne des quatre valeurs de fréquence obtenues en réa- lisant deux mesures sur chacune des deux joues de la mangue.

\subsection{Protocole expérimental}

Le poids et la densité du fruit ont été déterminés avant de procéder à l'évaluation de la fermeté de la mangue. Les coordonnées colorimétriques de la pulpe $(L, a, b)$ ont été mesurées à l'aide d'un chromamètre Minolta CR-300. La composition biochimique de chaque fruit a été analysée à partir d'un échantillon représentatif de la totalité de la pulpe du fruit. Le mode de préparation de cet échantillon a été différent selon le niveau de mûrissement du fruit. La pulpe amollie des fruits mûrs a été entièrement broyée au mixer (Waring) de manière à obtenir une pulpe homogène à partir de laquelle ont été prélevés les échantillons destinés à la détermination de l'extrait sec total, des sucres totaux et de l'amidon. La pulpe ferme des fruits immatures n'a pu être broyée à l'état frais aussi finement que celle des fruits mûrs. Afin de garantir une bonne représentativité de l'échantillon, la pulpe de ces fruits a été découpée en tranches fines. Celles-ci ont été congelées à $-20{ }^{\circ} \mathrm{C}$, puis lyophilisées et, après dessiccation complète, finement broyées au mixer Thermomix TM21 (Vorwerk). La dessiccation de la poudre de lyophilisat homogène ainsi obtenue a été achevée par étuvage sous vide à $70^{\circ} \mathrm{C}$ jusqu'à poids contant. Les échantillons lyophilisés ont été utilisés pour l'analyse des sucres totaux et de l'amidon.

L'extrait sec de la pulpe de mangue a été déterminé par pesée après déshydratation d'un échantillon de pulpe dans une étuve sous vide à $70^{\circ} \mathrm{C}$ jusqu'à poids constant. La prise d'essai a été d'environ $10 \mathrm{~g}$ pour un échantillon frais de pulpe de fruits mûrs et de (2 à 3) g pour les échantillons lyophilisés de pulpe des fruits immatures.

Les teneurs en glucose, fructose et saccharose de la pulpe ont été mesurées par chromatographie ionique (Dionex DX600) sur une colonne échangeuse d'anions (Carbopac PA1). Les composés ont été élués par une solution $\mathrm{NaOH} 50$ mM. La détection est alors assurée par ampérométrie pulsée (PED50, Dionex) selon la méthode décrite par Peschet et Giacalone [24]. L'extraction a 
Tableau I. Caractéristiques physico-chimiques de 133 mangues analysées (variétés Amélie et Keitt).

\begin{tabular}{|c|c|c|c|c|c|c|c|c|c|c|}
\hline \multirow[t]{3}{*}{ Variété } & \multicolumn{4}{|c|}{ Caractéristique du fruit entier } & \multicolumn{6}{|c|}{ Caractéristique de la pulpe } \\
\hline & \multirow{2}{*}{$\begin{array}{l}\text { Poids } \\
\text { (g) }\end{array}$} & \multirow{2}{*}{$\begin{array}{l}\text { Densité } \\
\left(\mathrm{kg} \cdot \mathrm{m}^{-3}\right)\end{array}$} & \multicolumn{2}{|c|}{ Fermeté } & \multirow{2}{*}{$\begin{array}{l}\text { Extrait sec } \\
\left(\mathrm{g} \cdot 100 \mathrm{~g}^{-1}\right)\end{array}$} & \multirow{2}{*}{$\begin{array}{l}\text { Sucres totaux } \\
\left(\mathrm{g} \cdot 100 \mathrm{~g}^{-1}\right)\end{array}$} & \multirow{2}{*}{$\begin{array}{l}\text { Amidon } \\
\left(\mathrm{g} \cdot 100 \mathrm{~g}^{-1}\right)\end{array}$} & \multicolumn{3}{|c|}{ Coordonnée colorimétrique } \\
\hline & & & $E^{1}(\mathrm{MPa})$ & $F 5^{2}(\mathrm{~N})$ & & & & $L$ & $a$ & $b$ \\
\hline \multicolumn{11}{|c|}{ Amélie (49 fruits) } \\
\hline Moyenne & 390 & 988 & 0,92 & 26,8 & 16,7 & $7,1^{4}$ & 1,21 & 76,4 & 1,2 & 51,1 \\
\hline Maximum & 488 & 1044 & 2,65 & 59,3 & 21,3 & $15,8^{4}$ & 5,87 & 86,0 & 10,3 & 66,9 \\
\hline Minimum & 311 & 959 & 0,26 & 6,0 & 11,5 & $3,6^{4}$ & 0,07 & 63,3 & $-5,0$ & 38,9 \\
\hline $\mathrm{Cv}^{3}(\%)$ & 11,7 & 1,8 & 68,1 & 63,1 & 16,8 & 32,6 & 120,1 & 7,8 & 340 & 14,4 \\
\hline \multicolumn{11}{|c|}{ Keitt (84 fruits) } \\
\hline Moyenne & 481 & 994 & 1,06 & 18,3 & 17,7 & 11,0 & 0,96 & 70,7 & $-3,3$ & 43,8 \\
\hline Maximum & 685 & 1026 & 3,41 & 64,4 & 20,3 & 15,3 & 8,57 & 85,8 & 0,6 & 56,4 \\
\hline Minimum & 328 & 930 & 0,34 & 6,0 & 15,5 & 2,9 & 0,04 & 48,5 & $-6,3$ & 30,7 \\
\hline $\mathrm{Cv}^{3}(\%)$ & 15,6 & 1,5 & 63,3 & 80,0 & 7,4 & 22,4 & 161 & 13,1 & 50,2 & 12,0 \\
\hline \multicolumn{11}{|c|}{ Amélie + Keitt } \\
\hline Moyenne & 447 & 992 & 1,01 & 21,5 & 17,3 & 10,1 & 1,06 & 72,9 & $-1,6$ & 46,6 \\
\hline Maximum & 685 & 1044 & 3,41 & 64,4 & 21,3 & 15,8 & 8,57 & 86,0 & 10,3 & 66,9 \\
\hline Minimum & 311 & 930 & 0,26 & 6,0 & 11,5 & 2,9 & 0,04 & 48,5 & $-6,3$ & 30,7 \\
\hline $\mathrm{Cv}^{3}(\%)$ & 17,5 & 1,6 & 64,8 & 80,0 & 11,7 & 29,0 & 143,3 & 11,7 & $-223,3$ & 15,1 \\
\hline \multicolumn{11}{|c|}{${ }^{1} E=$ coefficient d'élasticité du fruit. } \\
\hline \multicolumn{11}{|c|}{${ }^{2}$ F5 = force développée à $5,5 \mathrm{~mm}$ de profondeur. } \\
\hline${ }^{3} \mathrm{Cv}=\mathrm{coe}$ & le varia & & & & & & & & & \\
\hline
\end{tabular}

été réalisée à chaud, sous reflux, à l'aide d'une solution d'éthanol à $80 \%(\mathrm{v} / \mathrm{v})$. L'extrait, ajusté à $200 \mathrm{~mL}$ avec de l'eau déminéralisée, a été filtré sur membrane de porosité $0,45 \mu \mathrm{m}$ puis analysé. Les résultats ont été exprimés en $g$ de sucres totaux pour $100 \mathrm{~g}$ de matière fraîche.

L'amidon de la pulpe a été déterminé après séchage à $60^{\circ} \mathrm{C}$ et homogénéisation du résidu d'extraction insoluble à l'alcool, obtenu à l'issue de l'extraction des sucres. L'amidon contenu dans une fraction aliquote du résidu a été hydrolysé par voie acide (solution $0,15 \mathrm{M} \mathrm{HCl}$ à $105^{\circ} \mathrm{C}$, pendant $3 \mathrm{~h}$ ), puis enzymatique (milieu tamponné à $\mathrm{pH}=$ 4,8 à $60^{\circ} \mathrm{C}$, pendant $30 \mathrm{~min}$ ) par l'amyloglucosidase lyophilisée $\left(75 \mathrm{U} \cdot \mathrm{mg}^{-1}\right)$. Le glucose libéré a été dosé par chromatographie ionique. La teneur en amidon a été exprimée en $\mathrm{g}$ pour $100 \mathrm{~g}$ de matière fraîche.

\section{Résultats et discussion}

\subsection{Caractéristiques physico-chimiques des fruits}

L'analyse des caractéristiques physico-chimiques des lots de mangues montre que, pour l'ensemble des fruits analysés, la fermeté pénétrométrique $F 5$ a été comprise entre $(6,0$ et 64,4$) \mathrm{N}$ (tableau 1 ). Ces valeurs correspondent respectivement à des fruits mûrs consommables (pulpe amollie) et à des fruits immatures non consommables (pulpe ferme). Les valeurs maximales de fermeté pénétrométrique, observées pour les deux lots de mangues analysés, doivent cependant être comparées aux valeurs de fermeté obtenues par Mizrach et al. [16] sur des fruits fraîchement cueillis $(120 \mathrm{~N}<F 5<130 \mathrm{~N})$. Une partie non négligeable de la phase de 
maturation et de mûrissement du fruit, durant la période de transport et de commercialisation, n'a donc pas été prise en compte par notre étude du fait de l'origine des approvisionnements.

La fermeté acoustique $E$ a varié de $(0,26$ à 3,41) $\mathrm{MPa}$. Les fruits les moins avancés en maturité ont fourni les valeurs les plus élevées de ce coefficient d'élasticité.

Les lots de mangues analysés ont présenté une grande hétérogénéité de fermeté, que celle-ci soit mesurée par la méthode acoustique (coefficient de variation de 68,1\% pour Amélie et de 63,3\% pour Keitt) ou par pénétrométrie (coefficient de variation de 63,1 \% pour Amélie et de 80 \% pour Keitt).

Les teneurs en sucres totaux et en amidon mesurées dans la pulpe des fruits ont été conformes aux valeurs citées dans la littérature [7-9]. À l'instar des paramètres de fermeté $F 5$ et $E$, la teneur en amidon et la composante rouge $(a)$ de la couleur de la pulpe ont présenté une variabilité intra lot très élevée. À l'opposé, la variabilité intra lot des variables poids, densité, extrait sec ainsi que la luminance $(L)$ et la composante jaune $(b)$ de la couleur de la pulpe a été relativement faible. La variabilité des sucres totaux a été intermédiaire.

Les corrélations entre les variables ont été recherchées pour les fruits de chacune des deux populations Amélie et Keitt, et pour l'ensemble des fruits analysés, toutes variétés confondues (tableau II).

Pour la variété Amélie, la luminance $L$ se révèle relativement bien corrélée aux variables $F 5, E$ et à la teneur en amidon de la pulpe $(r=0,79, r=0,80$ et $r=0,68$, respectivement). Les niveaux de corrélation diminuent pour la variété Keitt $(r=0,64, r=0,60$ et $r=0,60$, respectivement) et pour la population rassemblant les fruits des deux variétés $(r=0,68, r=0,59$ et $r=0,59$, respectivement).

La composante rouge ( $a$ ) a présenté quant à elle une bonne corrélation avec les variables $F 5$ et $E$ pour les populations de mangues des variétés Amélie $(r=-0,69$ et $r=$ $-0,68$, respectivement ) et Keitt ( $r=-0,70$ et $r=-0,67$, respectivement). En revanche, les corrélations mesurées sur l'ensemble des fruits, toutes variétés confondues, ont fortement diminué ( $r=-0,28$ pour $F 5$ et $\mathrm{r}=-0,53$ pour E). Ce résultat confirme le caractère spécifique que constitue la couleur de la pulpe pour une variété donnée, ainsi que la spécificité de l'évolution de cette composante rouge (a) tout au long du processus de mûrissement du fruit après cueillette.

\subsection{Relation entre mesure des fermetés acoustique et pénétrométrique du fruit entier}

L'évolution de la fermeté acoustique $E$ a été étudiée en fonction de la fermeté pénétrométrique $F 5$ pour les 133 échantillons de mangue (figure 1). Le coefficient de corrélation obtenu pour ces 133 fruits $(r=0,81)$, toutes variétés confondues, est du même ordre de grandeur que celui obtenu pour les fruits de variété Amélie $(r=0,78)$. Il est en revanche nettement moins bon que celui observé pour les fruits de variété Keitt ( $r=$ 0,93 ) (tableau II). Cet effet variétal peut expliquer une partie de la dispersion des points expérimentaux, plus importante pour la variété Amélie que pour Keitt, en raison de l'influence de la forme du fruit et du rayon de courbure au point d'impact de la bille sur l'estimation de la fréquence de résonance et donc sur la fermeté acoustique $E$. Une telle explication avait déjà été donnée dans la littérature à propos des différences de coefficient de corrélation observées entre les paramètres de fermetés pénétrométrique et acoustique, pour différentes variétés de pommes [25, 26]. L'hétérogénéité de texture des mangues peut également contribuer à la dispersion des points expérimentaux (figure 1), la fermeté pénétrométrique F5 moyenne pour le fruit étant obtenue à partir de huit mesures locales uniformément réparties sur les deux joues. En présence de forts gradients de texture de la pulpe, l'estimation de $F 5$ peut s'avérer très imprécise et la valeur moyenne obtenue peut ne pas refléter le niveau global de fermeté du fruit.

\subsection{Relations entre la fermeté du fruit entier et la teneur en amidon de la pulpe}

La teneur en amidon de la pulpe s'est révélée bien corrélée aux deux paramètres de 
Tableau II.

Matrices de corrélation entre certains paramètres mesurant la qualité de lots de mangues de chacune des variétés Amélie et Keitt, ainsi que de l'ensemble des 133 mangues analysées.

\begin{tabular}{|c|c|c|c|c|c|c|c|c|c|}
\hline \multirow{2}{*}{$\begin{array}{l}\text { Paramètres } \\
\text { étudiés }\end{array}$} & \multirow[t]{2}{*}{ Densité } & \multirow[t]{2}{*}{ Extrait sec } & \multicolumn{3}{|c|}{ Coordonnées colorimétriques de la pulpe } & \multicolumn{2}{|c|}{ Paramètre de fermeté } & \multirow{2}{*}{ Amidon } & \multirow[t]{2}{*}{ Sucres totaux } \\
\hline & & & $L$ & $a$ & $b$ & $\begin{array}{c}\text { Pénétrométri } \\
\text { F5 }\end{array}$ & $\begin{array}{c}\text { Acoustique } \\
E\end{array}$ & & \\
\hline Densité & 1 & - & - & - & - & - & - & - & - \\
\hline Extrait sec & $0,44^{\star \star}$ & 1 & - & - & - & - & - & - & - \\
\hline$L$ & $-0,43^{\star \star}$ & 0,11 & 1 & - & - & - & - & - & - \\
\hline$a$ & $0,47^{\star \star}$ & 0,01 & $-0,90^{\star \star}$ & 1 & - & - & - & - & - \\
\hline$b$ & $0,40^{\star \star}$ & 0,29 & $-0,78^{\star *}$ & $0,76^{\star *}$ & 1 & - & - & - & - \\
\hline F5 & $-0,47^{\star \star}$ & $-0,17$ & $0,79^{\star *}$ & $-0,69^{\star *}$ & $-0,71^{\star *}$ & 1 & - & - & - \\
\hline$E$ & $-0,26$ & 0,32 & $0,80^{\star *}$ & $-0,68^{\star \star}$ & $-0,52^{\star \star}$ & $0,78^{\star \star}$ & 1 & - & - \\
\hline Amidon & 0,04 & $0,39^{\star *}$ & $0,68^{\star *}$ & $-0,50^{\star \star}$ & $-0,47^{\star \star}$ & $0,60^{\star \star}$ & $0,75^{\star \star}$ & 1 & - \\
\hline Sucres totaux & $-0,11$ & $0,59^{\star \star}$ & $0,52^{\star \star}$ & $-0,35$ & $-0,13$ & 0,47 & $0,54^{\star \star}$ & 0,26 & 1 \\
\hline \multicolumn{10}{|l|}{ Keitt } \\
\hline \multirow{2}{*}{$\begin{array}{l}\text { Paramètres } \\
\text { étudiés }\end{array}$} & \multirow[t]{2}{*}{ Densité } & \multirow[t]{2}{*}{ Extrait sec } & \multicolumn{3}{|c|}{ Coordonnées colorimétriques de la pulpe } & \multicolumn{2}{|c|}{ Paramètre de fermeté } & \multirow{2}{*}{ Amidon } & \multirow[t]{2}{*}{ Sucres totaux } \\
\hline & & & $L$ & $a$ & $b$ & $\begin{array}{c}\text { Pénétrométr } \\
\text { F5 }\end{array}$ & $\begin{array}{c}\text { Acoustique } \\
E\end{array}$ & & \\
\hline Densité & 1 & - & - & - & - & - & - & - & - \\
\hline Extrait sec & 0,18 & 1 & - & - & - & - & - & - & - \\
\hline$L$ & $-0,14$ & 0,03 & 1 & - & - & - & - & - & - \\
\hline$a$ & 0,02 & 0,01 & $-0,57^{\star \star}$ & 1 & - & - & - & - & - \\
\hline$b$ & 0,10 & $-0,05$ & $0,30^{\star *}$ & $0,45^{\star \star}$ & 1 & - & - & - & - \\
\hline F5 & 0,02 & 0,11 & $0,64^{\star *}$ & $-0,70^{\star \star}$ & $-0,23$ & 1 & - & - & - \\
\hline E & 0,11 & 0,11 & $0,60^{* *}$ & $-0,67^{\star \star}$ & $-0,13$ & $0,93^{\star \star}$ & 1 & - & - \\
\hline Amidon & 0,10 & 0,27 & $0,60^{\star \star}$ & $-0,60^{\star \star}$ & $-0,13$ & $0,88^{\star \star}$ & $0,89^{\star \star}$ & 1 & - \\
\hline Sucres totaux & 0,17 & 0,04 & $-0,34^{\star \star}$ & $0,42^{\star \star}$ & 0,17 & $-0,69^{\star \star}$ & $-0,70^{\star *}$ & $-0,71^{\star \star}$ & 1 \\
\hline \multicolumn{10}{|l|}{ Amélie + Keitt } \\
\hline \multirow{2}{*}{$\begin{array}{l}\text { Paramètres } \\
\text { étudiés }\end{array}$} & \multirow[t]{2}{*}{ Densité } & \multirow[t]{2}{*}{ Extrait sec } & \multicolumn{3}{|c|}{ Coordonnées colorimétriques de la pulpe } & \multicolumn{2}{|c|}{ Paramètre de fermeté } & \multirow{2}{*}{ Amidon } & \multirow[t]{2}{*}{ Sucres totaux } \\
\hline & & & $L$ & $a$ & $b$ & $\begin{array}{c}\text { Pénétrométri } \\
\text { F5 }\end{array}$ & $\begin{array}{c}\text { Acoustique } \\
E\end{array}$ & & \\
\hline Densité & 1 & - & - & - & - & - & - & - & - \\
\hline Extrait sec & $0,36^{\star \star}$ & 1 & - & - & - & - & - & - & - \\
\hline$L$ & $-0,27$ & $-0,02$ & 1 & - & - & - & - & - & - \\
\hline$a$ & 0,12 & $-0,13$ & $-0,24^{\star \star}$ & 1 & - & - & - & - & - \\
\hline$b$ & 0,13 & 0,02 & 0,10 & $0,75^{\star \star}$ & 1 & - & - & - & - \\
\hline F5 & $-0,24^{\star \star}$ & $-0,12$ & $0,68^{* *}$ & $-0,28^{\star *}$ & $-0,23$ & 1 & - & - & - \\
\hline$E$ & $-0,02$ & 0,22 & $0,59^{* *}$ & $-0,53^{\star *}$ & $-0,30^{\star *}$ & $0,81^{\star \star}$ & 1 & - & - \\
\hline Amidon & 0,06 & $0,28^{\star *}$ & $0,59^{\star *}$ & $-0,26^{\star *}$ & $-0,16$ & $0,76^{\star \star}$ & $0,83^{* *}$ & 1 & - \\
\hline Sucres totaux & $0,29^{\star \star}$ & $0,45^{\star \star}$ & $-0,39^{\star \star}$ & $-0,29^{\star \star}$ & $-0,09$ & $-0,62^{\star \star}$ & $-0,34^{\star \star}$ & $-0,49^{\star \star}$ & 1 \\
\hline
\end{tabular}


Figure 1.

Relation entre les paramètres de fermeté mesurés sur fruit entier au pénétromètre (F5) ou par la méthode acoustique impulsionnelle $(E)$, pour les variétés de mangues Keitt et Amélie.

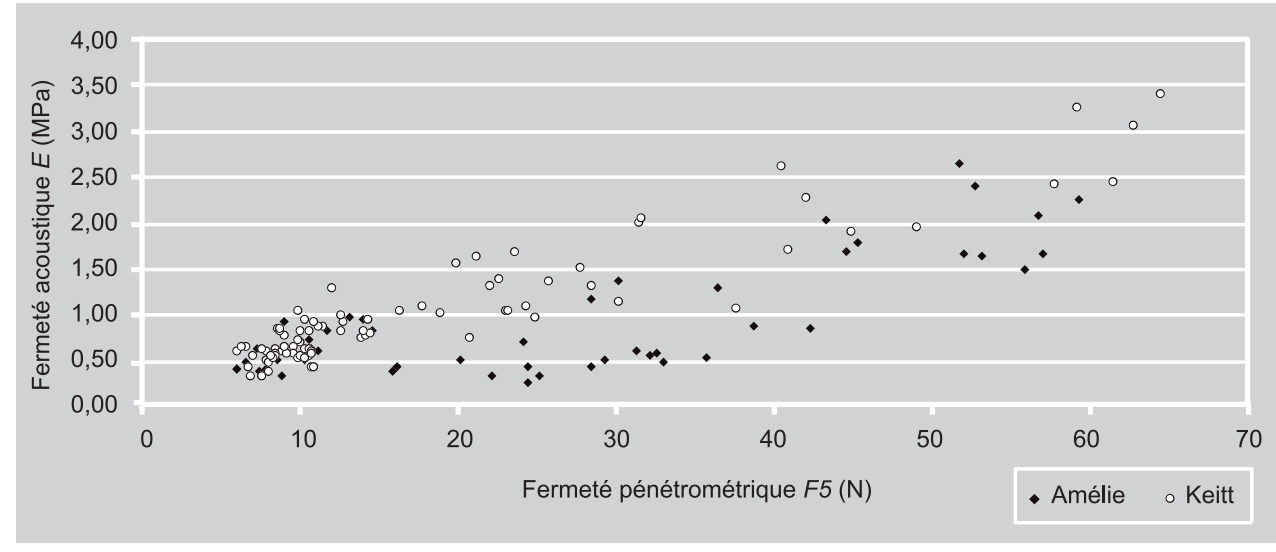

Figure 2.

Relation entre la fermeté pénétrométrique $(F 5)$ du fruit entier et la teneur en amidon de la pulpe pour les variétés de mangues Keitt et Amélie.

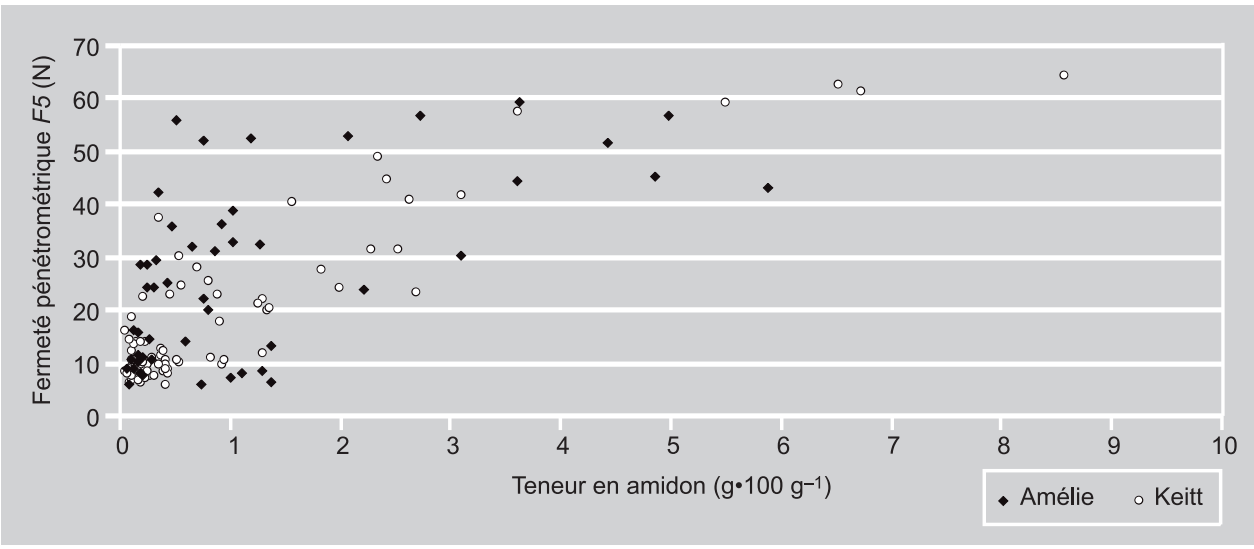

fermeté $E$ et $F 5$ mesurés sur fruit entier. Les meilleurs coefficients de corrélation ont été obtenus pour la population de mangues Keitt $(r=0,89$ pour $E, r=0,88$ pour $F 5)$ et pour la population rassemblant les fruits des deux variétés Amélie et Keitt $(r=0,83$ pour $E, r=0,76$ pour $F 5)$. La variété Amélie se distingue par une faible corrélation entre la teneur en amidon et la fermeté pénétrométrique $F 5(r=0,60)$, le coefficient d'élasticité $E$ demeurant encore relativement bien corrélé à la teneur en amidon $(r=0,75)$. Ces valeurs sont à comparer avec les corrélations obtenues par Rocha et al. [11] pour la variété Tommy Atkins entre la fermeté pénétrométrique et un indice de dégradation de l'amidon $(r=-0,87)$, cet indice étant lui même corrélé à la teneur en amidon de la pulpe $(r=-0,74)$.
L'évolution des deux paramètres de fermetés pénétrométrique $F 5$ et acoustique $E$ en fonction de la teneur en amidon de la pulpe (figures 2, 3) montre que la méthode acoustique semble être la mieux adaptée au suivi de la dégradation de l'amidon en cours de maturation du fruit. Outre le fait que la mesure de $E$ est rapide et non destructive, la dispersion observée pour les points expérimentaux est plus faible pour $E$ que pour $F 5$.

L'évolution de la fermeté acoustique $E$ de la mangue en fonction de la teneur en amidon $A$ de la pulpe, pour la population des 133 mangues des variétés Keitt et Amélie, est bien représentée par la droite de régression suivante : $E=(0,357 \cdot \mathrm{A})+0,628(r=0,83), A$, teneur en amidon, étant exprimé en $\mathrm{g} \cdot 100 \mathrm{~g}^{-1}$ de pulpe fraîche. L'erreur standard du modèle est de 0,37 $\mathrm{MPa}$. 


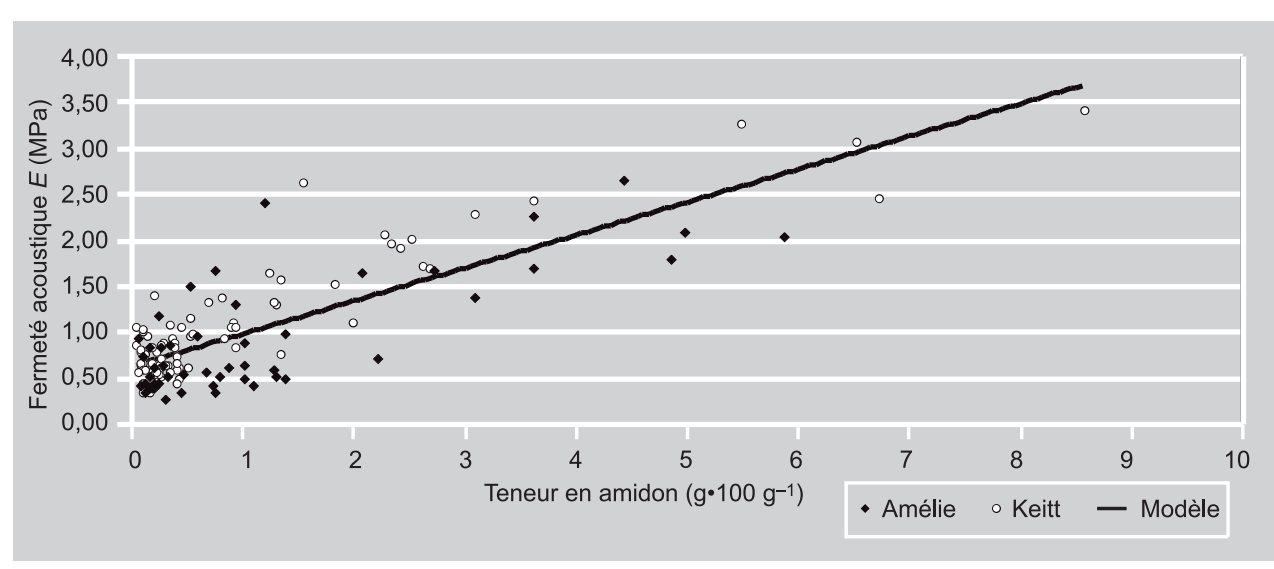

La dispersion des points expérimentaux par rapport au modèle (figure 3) pourrait résulter d'une évaluation imprécise de la fermeté acoustique $E$, d'une part, et de la teneur en amidon de la pulpe, d'autre part. Des études antérieures [21] ont montré que l'erreur d'estimation de la fermeté acoustique proviendrait essentiellement d'une détermination erronée de la fréquence de résonance des mangues, elle-même due soit à des variations locales importantes du rayon de courbure du fruit au point d'impact, soit au phénomène de modification d'amplitude des pics du spectre de fréquence du fruit. L'incertitude de mesure de la teneur en amidon de la pulpe fraîche de mangue, selon le protocole utilisé, a été estimée à $15 \%$. Cette valeur représente l'écart moyen de la teneur en amidon observé pour cinq échantillons de pulpe, issus de cinq fruits différents, analysés deux fois, à des dates différentes.

La dispersion observée peut également refléter un phénomène réel traduisant une variabilité de la fermeté des fruits, générée par la variation de paramètres de la pulpe autres que la teneur en amidon. L'état de dégradation des parois cellulaires, la pression osmotique, le volume intercellulaire et bien d'autres facteurs participent en effet à l'élaboration de la fermeté des fruits. Lors de la maturation, ces paramètres peuvent varier de façon concomitante, mais avec des cinétiques différentes, et être ainsi à l'origine des différences de fermeté observées pour des fruits par ailleurs d'égale teneur en amidon.
L'influence de ces paramètres de la pulpe sur la fermeté des fruits pourrait être d'autant plus sensible que les fruits analysés proviendraient de lots différents résultant de conditions agronomiques de production et de conservation variables selon les lots. Cette hypothèse est confortée par l'observation de meilleures corrélations entre la fermeté acoustique et la teneur en amidon pour les fruits Keitt que pour les fruits Amélie. En effet, les fruits de la variété Keitt proviennent tous d'un même lot initial de fruits, réceptionnés au laboratoire et conservés à $13^{\circ} \mathrm{C}$. À l'opposé, les mangues Amélie ont été achetés à intervalles de temps réguliers, sur une période de 4 semaines, donc sans garantie quant à leur provenance et date de récolte.

Le suivi de lots de fruits dont on maîtriserait à la fois le stade de maturité à la cueillette et la phase de maturation postrécolte apporterait vraisemblablement une réponse à cette interrogation sur la nature et les causes de cette dispersion.

Contrairement au test à l'iode, qui mesure le taux de régression de l'amidon à l'aide de valeurs ordinales, dépendantes du jugement humain, la technique acoustique impulsionnelle permet d'évaluer, sur une échelle continue et de manière objective, la teneur résiduelle en amidon. La méthode nécessite un étalonnage préalable spécifique donnant, pour chaque variété et vraisemblablement zone de production, la relation entre le coefficient d'élasticité $E$ et la teneur en amidon de la pulpe. Les corrélations obtenues entre
Figure 3.

Relation entre le coefficient d'élasticité $(E)$, mesuré par la méthode acoustique impulsionnelle, et la teneur en amidon de la pulpe pour les variétés de mangues Keitt et Amélie. 
la fermeté acoustique et la teneur en amidon de la chair sont d'autant plus hautement significatives que les fruits analysés ont été issus d'un même lot de départ. Lorsque ces conditions sont réunies, la mesure du coefficient d'élasticité $E$ peut conduire à une estimation de la teneur résiduelle de l'amidon du fruit aussi précise que celle obtenue avec un test destructif à l'iode.

De plus, à l'instar de la méthode de mesure de la fermeté acoustique, l'estimation de la teneur en amidon par la méthode acoustique impulsionnelle sera d'autant plus précise que cette estimation portera sur des fruits parvenus à un stade de maturation peu avancé, dont la pulpe ferme possède des propriétés viscoélastiques caractérisées par une composante élastique importante.

\section{Conclusions}

L'amidon, constituant majeur de la pulpe de la mangue au stade de la cueillette, participe fortement à l'élaboration de la texture de la pulpe et à la fermeté du fruit. La teneur en amidon de la pulpe est en effet bien corrélée aux deux paramètres de fermetés acoustique $E$ et pénétrométrique $F 5$, mesurés sur fruit entier. Les meilleurs coefficients de corrélation ont été obtenus pour la population de mangues de la variété Keitt $(r=0,89$ pour $E, r=0,88$ pour $F 5$ ). En revanche, pour la variété Amélie, la méthode acoustique de mesure de la fermeté semble être mieux adaptée au suivi de la dégradation de l'amidon que ne l'est la pénétrométrie. D’une manière générale, la précision de l'évaluation de la teneur en amidon est d'autant meilleure que les fruits sont à un stade de maturation peu avancé et possèdent une pulpe ferme.

La méthode acoustique impulsionnelle peut constituer un outil intéressant capable d'apprécier, de manière non destructive, le potentiel de conservation des fruits par l'évaluation concomitante de la fermeté globale du fruit et de la teneur résiduelle en amidon de sa pulpe. Le coefficient de corrélation mesuré entre le coefficient d'élasticité $E$ et la teneur en amidon diffère en fonction de la variété de mangue. Lorsque cette corré- lation est élevée, la technique acoustique impulsionnelle peut donner, indirectement, d'aussi bonnes informations sur l'état de dégradation de l'amidon dans le fruit en cours de conservation qu'une méthode destructive comme celle du test à l'iode.

\section{Remerciements}

Les auteurs remercient Marie Christine Laon et Jean Théophile Banzouzi pour leur assistance technique dans la réalisation de ce travail.

\section{Références}

[1] Gomez-Lim M.A., Postharvest physiology, in: Litz R.E. (Ed.), The mango, botany, production and uses, CAB International, Wallingford, UK, 1997, pp. 425-445.

[2] Mitra S.K., Baldwin E.A., Mango, in: Mitra S.K. (Ed.), Postharvest physiology and storage of tropical and subtropical fruits, CAB International, Wallingford, UK, 1997, pp. 85122.

[3] Johnson G.I., Sharp J.L., Milne D.L., Oosthuyse S.A., Postharvest technology and quarantine treatments, in: Litz R.E. (Ed.), The mango, botany, production and uses, CAB International, Wallingford, UK, 1997, pp. 447-507.

[4] Pruthi J.S., Harvesting: harvest maturity, maturity indices and harvesting techniques, in: Patnaik G. (Ed.), The mango book, production, post harvest technology and export marketing, Agric. \& Process. Food Products, Export Dev. Auth., New Delhi, India, Vol. I, 1992, p. 247.

[5] Kosiyachinda S., Lee S.K., Poernomo L., Maturity indices for harvesting of mango, in: Mendoza D.B., Wills R.B.H. (Eds.), Mango: fruit development, postharvest physiology and marketing in ASEAN, ASEAN Food Handling Bureau, Kuala Lumpur, Malaysia, 1984, pp. 33-36.

[6] Quintana E.G., Nanthachai P., Hiranpradit H., Mendoza D.B., Ketsa S., Changes in mango during growth and maturation, in: Mendoza D.B., Wills R.B.H. (Eds.), Mango: fruit development, postharvest physiology and marketing in ASEAN, ASEAN Food Handling Bureau, Kuala Lumpur, Malaysia, 1984, pp. 21-27. 
[7] Pantastico B., Lam P.F., Ketsa S., Yuniarti S., Kosittrakul M., Postharvest physiology and storage of mango, in: Mendoza D.B., Wills R.B.H. (Eds.), Mango: fruit development, postharvest physiology and marketing in ASEAN, ASEAN Food Handling Bureau, Kuala Lumpur, Malaysia, 1984, pp. 39-53.

[8] Hulme A.C., The mango, in: Hulme A.C. (Ed.), The biochemistry of fruits and their products, Acad. Press, New York, USA, 1971.

[9] Vasquez-Salinas C., Lakshminarayana S., Compositional changes in mango fruit during ripening at different storage temperatures, J. Food Sci. 50 (1985) 1646-1648.

[10] Roy B.N., Biswas S., Studies on maturity standard of mango (Mangifera indica L.) cv. Bombai Orissa, J. Hortic. 9 (1) (1981) 7-9.

[11] Rocha R.H.C., Menezes J.B., De Morais E.A., Da Silva G.G., Ambrosio M.M., Alvez M.Z., Use of starch degradation index for determining maturity in mango Tommy Atkins, Rev. Bras. Frutic. 23 (2001) 302-305.

[12] Peirs A., Scheerlinck N., Perez A.B., Jancsok P., Nicolaï B., Uncertainty analysis and modelling of the starch index during apple fruit maturation, Postharvest Biol. Technol. 26 (2002) 199-207.

[13] Saranwong S., Sornsrivichai J., Kawano S., Prediction of ripe-stage eating quality of mango fruit from its harvest quality measured nondestructively by near infrared spectroscopy, Postharvest Biol. Technol. 31 (2004) 137-145.

[14] Tridjaja N.O., Mahendra M.S., Maturity indices and harvesting practice of Arumanis mango related to the target market, in: Johnson G.I., Le Van T., Nguyen Duy D., Webb M.C. (Eds.), Quality assurance in agricultural produce, ACIAR Proc. 100, Canberra, Australia, 2000, pp. 129-133.

[15] Baez-Sanudo R., Siller-Cepeda J., Bringas T.E., Baez S.M., Determination of maturity indices in the main mango cultivars produced in Mexico, in: Proc. Interam. Soc. Trop. Hortic., 1993, pp. 148-154.
[16] Mizrach A., Flitsanov U., Fuchs Y., An ultrasonic nondestructive method for measuring maturity of mango fruit, Trans. ASAE 40 (1997) 1107-1111.

[17] Peleg M., Evaluation by instrumental methods of the texture and properties of some tropical fruit, J. Texture Stud. 10 (1979) 45-65.

[18] Polderdijk J.J., Kho R.M., Carmi Y., De Kruif A.P., Firmness of mango (Mangifera indica L.) measured acoustically, Acta Hortic. 509 (2000) 861-865.

[19] Mizrach A., Flitsanov U., Schmilovitch Z., Fuchs Y., Determination of mango physiological indices by mechanical wave analysis, Postharvest Biol. Technol. 16 (1999) 179186.

[20] Shmulevich I., Galili N., Benichou N., Development of a non destructive method for measuring the shelf-life of mango fruit, Proc. FPAC IV Conf., ASAE, Chicago, USA, 1995, pp. 275-287.

[21] Valente M., Banzouzi J.T., Dornier N., Évaluation non destructive de la fermeté de la mangue par la technique acoustique impulsionnelle, Fruits 55 (2000) 333-345.

[22] Cooke J.R., An interpretation of the resonant behavior of intact fruits and vegetables, Trans. ASAE 15 (1972) 1075-1080.

[23] Duprat F., Grotte M., Pietri E., Loonis D., The acoustic impulse response method for measuring the overall firmness of fruit, J. Agric. Eng. Res. 66 (1997) 251-259.

[24] Peschet J.L., Giacalone A., Un nouveau concept en analyse des sucres : la chromatographie ionique couplée à l'ampérométrie pulsée, Ind. Agric. Aliment. 108 (1991) 1-4.

[25] Abbott J.A., Massie D.R., Upchurch B.L., Hruschka W.R., Nondestructive sonic firmness measurement of apples, Trans. ASAE 38 (5) (1995) 1461-1466.

[26] De Belie N., Schotte S., Coucke P., De Baerdemaeker J., Development of an automated monitoring device to quantify changes in firmness of apples during storage, Postharvest Biol. Technol. 18 (2000) 1-8. 


\section{Relación entre la firmeza del mango fresco y el contenido de almidón de la pulpa.}

Resumen - Introducción. La firmeza del fruto y el contenido de almidón de la pulpa son dos criterios fisicoquímicos de gran interés para un fruto climatérico como el mango. Representan dos buenos indicadores de la madurez de los frutos en la recolección y de su grado de maduración durante la conservación. Por lo que sabemos, existen pocos estudios que relacionen el contenido de almidón de la pulpa, principal compuesto del mango en la fase de recolección, y la firmeza del fruto. Material y métodos. Se aplicaron dos métodos de evaluación de la firmeza del fruto entero: un método penetrométrico destructivo que permite conocer la fuerza utilizada para penetrar en el fruto hasta una profundidad de $5,5 \mathrm{~mm}$ (F5), y un método acústico de impulsos no destructivo que permite determinar un coeficiente global de elasticidad $(E)$ del fruto. El contenido de almidón se determinó en una muestra, representativa de la pulpa entera del fruto, tratada por hidrólisis ácida y, luego, enzimática. La glucosa liberada fue dosificada mediante cromatografía iónica. Se realizaron ensayos en un total de 133 frutos, pertenecientes a dos poblaciones de mangos de las variedades Keitt y Amélie y abarcando una amplia gama de maduraciones. Resultados y discusión. El contenido de almidón de la pulpa mostró una buena correlación con los parámetros de firmeza $F 5$ y $E$ medidos en frutos enteros. Los mejores coeficientes de correlación se obtuvieron en la población de mangos Keitt ( $r=0,89$ para $E ; r=0,88$ para $F 5)$ y en la población que reunía al conjunto de frutos de ambas variedades $(r=0,83$ para $E ; r=0,76$ para F5). La variedad Amélie se distingue por un bajo coeficiente de correlación entre el contenido de almidón y la firmeza penetrométrica $F 5(r=0,60)$, el coeficiente de elasticidad $E$ sigue estando relativamente bien correlacionado con el contenido de almidón $(r=0,75)$. Conclusión. El método acústico de impulsos puede constituir una herramienta interesante capaz de evaluar, de manera no destructiva, el potencial de conservación de los frutos mediante la evaluación concomitante de la firmeza global del fruto y el contenido residual de almidón de su pulpa. El coeficiente de correlación observado entre el coeficiente de elasticidad $E$ y el contenido de almidón difiere según la variedad de mango. Cuando este coeficiente de correlación es alto, la técnica acústica de impulsos puede dar, indirectamente, una información tan buena sobre el nivel de degradación del almidón en el fruto en conservación como un método destructivo como el de la prueba del yodo.

Francia / Mangifera indica / frutas / firmeza / almidón / métodos no destructivos / medicon / penetrómetros

To access this journal online: www.edpsciences.org 